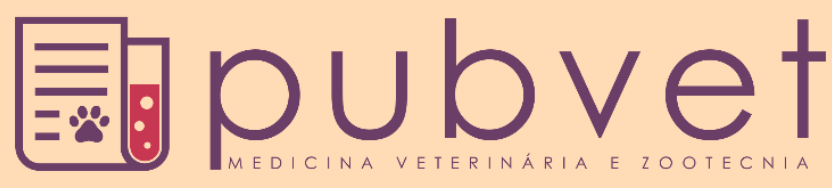

https://doi.org/10.31533/pubvet.v13n8a393-6

\title{
Distocia em calopsita (Nymphicus hollandicus) com complicações metabólicas por isquemia renal: relato de caso
}

\author{
Átilla Holanda de Albuquerque ${ }^{1 * \bullet}$, Ingrid Rabelo Rodrigues ${ }^{2} \bullet$, Elisângela de Souza \\ Lopes $^{1}{ }^{\circ}$, Rosa Patrícia Ramos Salles ${ }^{3}$, Isaac Neto Goes da Silva ${ }^{\circ}$, Régis Siqueira de \\ Castros Teixeira1, William Cardoso Maciel $^{50}$, Michelle Costa e Silva ${ }^{6}{ }^{\circ}$ \\ ${ }^{I}$ Dr(a). em Ciências Veterinárias, Universidade Estadual do Ceará, Fortaleza, CE. \\ ${ }^{2}$ Acadêmico de Medicina Veterinária, Faculdade Terra Nordeste, Estagiário do Hospital Veterinário Metropolitano, Caucaia, CE. \\ ${ }^{3}$ Dra. em Ciências Veterinárias, Médica Veterinária, Biolab Clínica e Laboratório Veterinário LTDA, Fortaleza, CE, Brasil. \\ ${ }^{4}$ Prof. Dr., Patologia Clínica Veterinária, Universidade Estadual do Ceará, Fortaleza, CE. Brasil. \\ ${ }^{5}$ Prof. Dr., Adjunto, Ornitopatologia, Universidade Estadual do Ceará, Fortaleza, CE, Brasil. \\ ${ }^{6}$ Profa. Msc., Patologia Clínica Veterinária, Faculdade Terra Nordeste, Caucaia, CE. Brasil. \\ * Autor para correspondência, E-mail: atillaholanda@hotmail.com
}

Resumo. A distocia em psitacideos é comum, sendo definida como uma obstrução do trato reprodutivo por um ou mais ovos, devido a problemas em sua formação, como também poder ser influenciada por fatores como os físicos, nutricionais e genéticos, entre outros. $\mathrm{O}$ presente trabalho objetivou relatar o caso clínico de uma calopsita (Nymphicus hollandicus) com aproximadamente dois anos de idade apresentando complicações isquêmicas renais secundárias à prolongada retenção de ovo, atendida no Hospital Veterinário Metropolitano do município de Caucaia - Ceará, diagnosticada com distocia após anamnese, exame físico e radiografia abdominal. Após confirmação da afecção, foi realizado tratamento de suporte seguido de ovocentese, no entanto, o paciente veio a óbito em 48 horas. Na necropsia, observou-se, o quadro de comprometimento metabólico em virtude do acúmulo dos ovos na cavidade, favorecendo a ruptura de oviduto, aderência e isquemia de outros órgãos.

Palavras-chave: celiotomia, nefropatia, retenção de ovo, terapia conservativa

\section{Dystocia in Nymphicus hollandicus with metabolic complications due to renal ischemia: case report}

\begin{abstract}
Dystocia in psitacides is common, being defined as an obstruction of the reproductive tract by one or more eggs, due to problems in its formation, as well as to be influenced factors such as physical, nutritional, and genetic among others. The present study aimed to report a clinical case of a female Nymphicus hollandicus, with about twoyear-old presenting with ischemic renal complications secondary to prolonged maintenance, attended at the Hospital Metropolitan veterinarian of the municipality of Caucaia - Ceará, diagnosed with dystocia after anamnesis, physical examination and radiography abdominal. After contamination of the condition, treatment was established support followed hours observes three eggs free in the celtic cavity, oviduct rupture, egg adherence medium with the intestine, and renal ischemia. It can be concluded that in cases of dystocia associated with late treatment there is a greater possibility of occurrence of metabolic impairment due to the accumulation of eggs in the cavity, favoring the rupture of oviduct, adhesion and ischemia of other organs.
\end{abstract}

Key words: celiotomy, nephropathy egg retention, conservative therapy 


\section{Distocia en Nymphicus hollandicu con complicaciones metabólicas debido a isquemia renal: reporte de un caso}

Resumen. La distocia en psitácidos es común, se define como una obstrucción del tracto
reproductivo por uno o más huevos, debido a problemas en su formación, así como
influencia de factores físicos, nutricionales y genéticos, entre otros. El presente estudio
tuvo como objetivo informar sobre el caso clínico de una Nymphicus hollandicus con edad
de aproximadamente dos años, la misma presentaba complicaciones isquémicas renales
secundarias a la retención prolongada de huevos. El caso fue atendido en el Hospital
Veterinario Metropolitano de Caucaia - Ceará, diagnosticada con distocia después de una
anamnesis, exploración física y radiografía abdominal. Posteriormente a la confirmación
de la condición, se realizó un tratamiento de apoyo seguido de ovocentesis, sin embargo,
el paciente falleció a las 48 horas. En la necropsia, se observó el cuadro relacionado al
comprometimiento metabólico causado por la acumulación de huevos en la cavidad,
favoreciendo la ruptura del oviducto, la adhesión y la isquemia de otros órganos.

Palabras clave: celiotomía, nefropatía, retención de óvulos, terapia conservadora

\section{Introdução}

Na medicina de aves, patologias no trato reprodutivo de fêmeas são bem comuns (Rosen, 2012). Dentre elas a distocia, definida com um processo patológico por uma obstrução do trato reprodutivo devido à presença de um ou mais ovos, com complicações devido a problemas uni ou multifatoriais como a má formação dos mesmos, tamanho e forma, consistência e superfície da casca, aumento na produção dos ovos, atonia uterina, predisposição genética, deficiências metabólicas, ocorrência de infecções, erros de manejo como nutrição inadequada, obesidade e ou condições de estresse, tumor no trato reprodutivo, ou compressão do mesmo por massas abdominais, dentre outros (Castro et al., 2013; Crosta et al., 2003; Matos \& Morrisey, 2005; Rosen, 2012). A patologia é bastante comum em psitacídeos como calopsitas e agapornis, ocorrendo ainda em alguns passeriformes, como nos pintassilgos e canários, observando-se casos também nas demais aves (Castro et al., 2013; Rosen, 2012).

Aves com patologias reprodutivas podem apresentar desde sinais clínicos brandos até quadros de choque. Aves acometidas com distocia podem apresentar quadros de apatia, dispneia, diarreia ou aquesia, oviduto inferior distendido com distensão celômica, inquietação, tensão muscular, relutância em se movimentar, claudicação, paresia e morte súbita (Gündüz et al., 2009; Rosen, 2012).

O diagnóstico é guiado por suspeita clínica durante a anamnese, histórico, por palpação do abdômen durante o exame físico, com atenção a casos de presença de ovos com casca mole, os quais podem não ser palpáveis, no entanto, podem ser observados em casos de dilatação do colón. Como diagnóstico definitivo utiliza-se radiografia e a ultrassonografia, que podem revelar um ovo com aumento de densidade e a posição do mesmo (Crosta et al., 2003; Hasholt, 1966; Rosen, 2012).

O tratamento conservativo de suporte envolve o controle térmico, oxigenoterapia, fluidoterapia, suporte nutricional, analgesia e dependendo da causa primária utiliza-se gluconato de cálcio por via intramuscular (Crosta et al., 2003). Em casos de perfuração do trato reprodutivo, com presença de celiomite supurativa, é indicada a antibioticoterapia. Também pode ser aplicado prostaglandina local para relaxar o esfíncter urovaginal, facilitando a passagem do ovo, prevenindo a ruptura das glândulas, que ocorre quando o esfíncter permanece fechado. Para promoção de contrações da musculatura uterina também pode ser utilizada ocitocina, a fim de auxiliar na expulsão do ovo (Gündüz et al., 2009; Rosen, 2012).

Não ocorrendo a ovoposição após o tratamento medicamentoso, devem ser realizadas manobras de remoção manual com pressão digital, e em caso de insucesso destas, a ovocentese, a qual pode ser feita pela cloaca ou através da parede abdominal(Gündüz et al., 2009), utilizando uma agulha calibrosa introduzida no ovo, sendo seu conteúdo aspirado e, posteriormente aplicada uma pequena compressão para que o ovo colabe (Hasholt, 1966). A celiotomia também é outra ferramenta cirúrgica citada na literatura, indicada nos casos onde o ovo não pode ser visualizado pela cloaca, utilizando-se as técnicas de histerotomia, para a remoção de ovos retidos, cascas e restos dos mesmos, ou histerectomia, indicada 
em casos de postura crônica e retenção recorrente, patologias no oviduto e presença de tumores (Castro et al., 2013; Hasholt, 1966).

Objetivou-se relatar o caso clínico de uma calopsita (Nymphicus hollandicus) com complicações isquêmicas secundárias a prolongada retenção de ovo.

\section{Relato de caso}

Foi atendida no Hospital Veterinário Metropolitano (HVM), uma Calopsita (Nymphicus hollandicus) fêmea, com idade aproximada de dois anos, pesando 80 gramas. Durante a anamnese o proprietário relatou que a ave se alimentava de sementes diversas, principalmente girassol, vivia pareada com um macho e criados dentro da residência com luminosidade indireta. Também foi relatado que a ave apresentou um quadro de distocia, tratado pelo proprietário inicialmente com massagem abdominal, administração de unguento e compressa de água morna, com sucesso de postura do ovo após 2 horas. Após 48 horas, a ave apresentou um novo quadro distocia, o proprietário novamente utilizou a mesma técnica supracitada por um período de cinco dias, sem nenhum sucesso de postura, o tutor procurou atendimento clínico veterinário, onde inicialmente foi realizado um tratamento medicamentoso desconhecido pelo proprietário (durante a consulta), e solicitado um exame radiográfico. No décimo primeiro dia de distocia, o paciente foi atendido no HVM apresentando quadro de apatia, anorexia, adipsia e aumento de volume abdominal.

Durante o exame físico, foi observado volume rígido e esférico no abdômen, desidratação, atrofia muscular, cianose e fezes com quantidade de uratos significante, no entanto com ausência de dificuldade respiratória. Na radiografia apresentada foi observada a presença de ovo de casca espessada e de maior radiopacidade em terço distal da cavidade celomática, medindo $3,28 \times 1,86 \mathrm{~cm}$, sugerindo deslocamento cranial dos órgãos abdominais (Figura 1), imagem compatível com retenção de ovo, causando distocia na ave (Rosen, 2012).
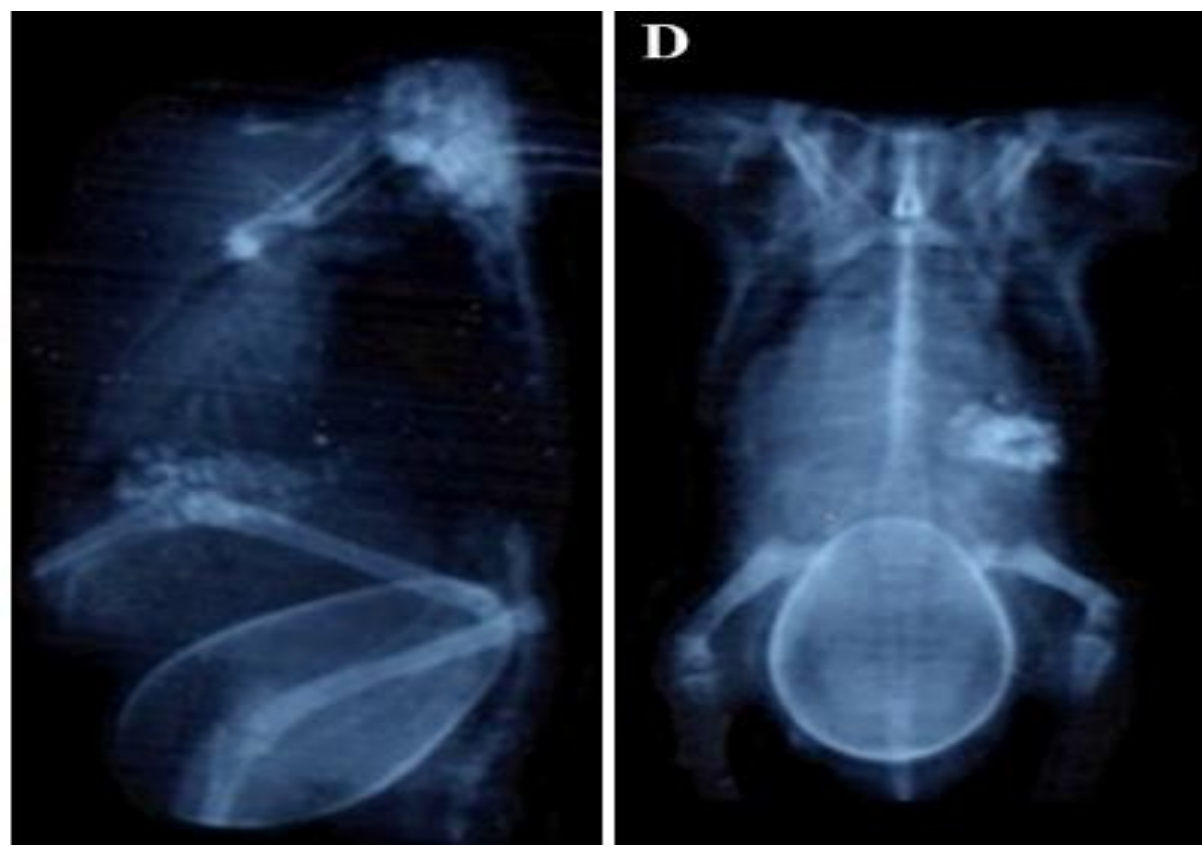

Figura 1. Exame radiográfico do paciente com nove dias de distocia, com presença de ovo de casca espessada e de maior radiopacidade em terço distal da cavidade celomática.

O paciente foi internado para reestabelecer o quadro de hidratação com fluidoterapia por via subcutânea utilizando solução de ringer com lactato $(2 \mathrm{~mL}$ da solução a cada $6 \mathrm{~h})$, com nutrição via oral (galvagem) utilizando sonda diretamente no papo sendo $1,5 \mathrm{~mL}$ a cada 3 horas, e também foi administrado gluconato de cálcio na dose de $0,02 \mathrm{~mL} / \mathrm{g}$ de solução a $1 \%$ por via intramuscular. Após 12 horas de administração de gluconato de cálcio sem sucesso de postura, foi realizada a ovocentese (Figura $2 \mathrm{~A}$ e $2 \mathrm{~B}$ ), utilizando agulha $40 \times 12 \mathrm{~mm}$ com única incisão drenando aproximadamente $2 \mathrm{~mL}$ de conteúdo do ovo. 
Seguidamente, foi administrado enrofloxacina na dose de $15 \mathrm{mg} / \mathrm{kg}$ por via intramuscular a cada 24 horas como terapia antimicrobiana, administrou-se meloxican na dose de $0,5 \mathrm{mg} / \mathrm{kg}$ por via intramuscular como terapia anti-inflamatória, e devido o quadro de concentração de uratos nas fezes (Figura 2C), foi administrado alopurinol na dose $2 \mathrm{mg} / \mathrm{kg}$ por via oral a cada 8 horas, como um inibidor da xantina oxidase.
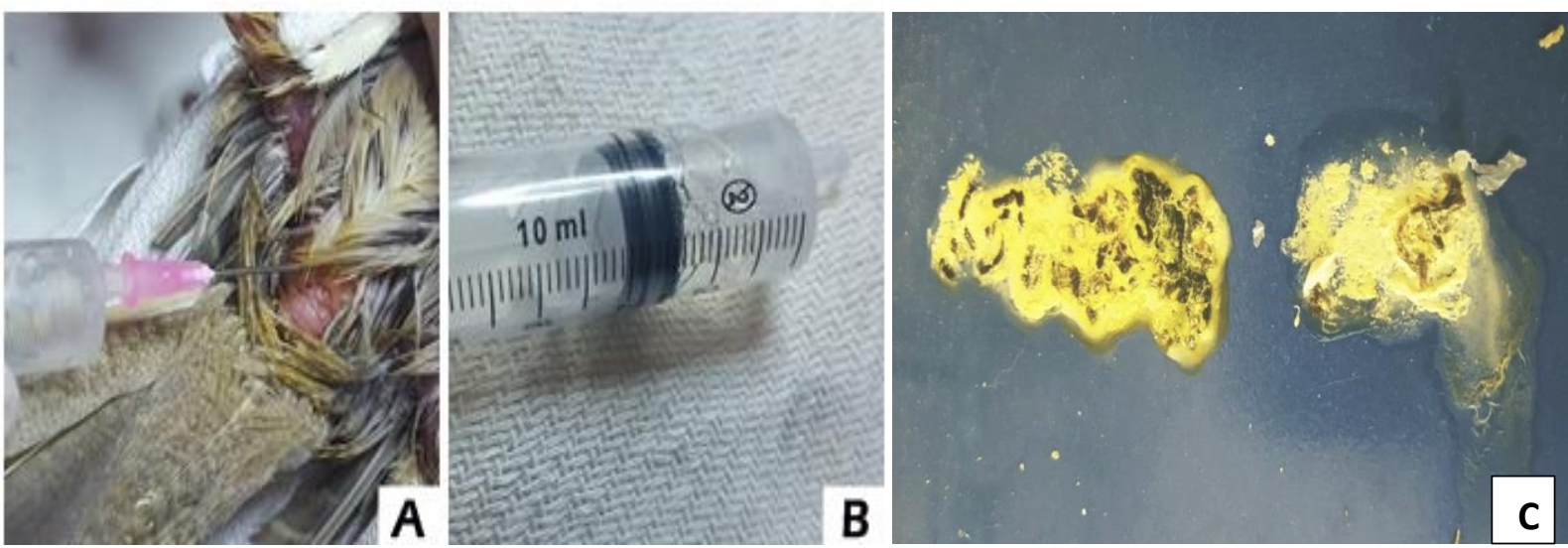

Figura 2. A) Procedimento de ovocentese através da parede abdominal; B) Conteúdo do ovo aspirado com volume aproximado de $2 \mathrm{~mL}$; C) Imagem macroscópica das fezes, demonstrando a grande quantidade de excreção de uratos.

No entanto, a ave veio a óbito após 48 horas do procedimento de ovocentese, sendo realizada necropsia (Figura 3), identificando a presença de três ovos livres na cavidade celomática, com ruptura de oviduto, aderência intestinal no ovo médio e presença de isquemia renal.
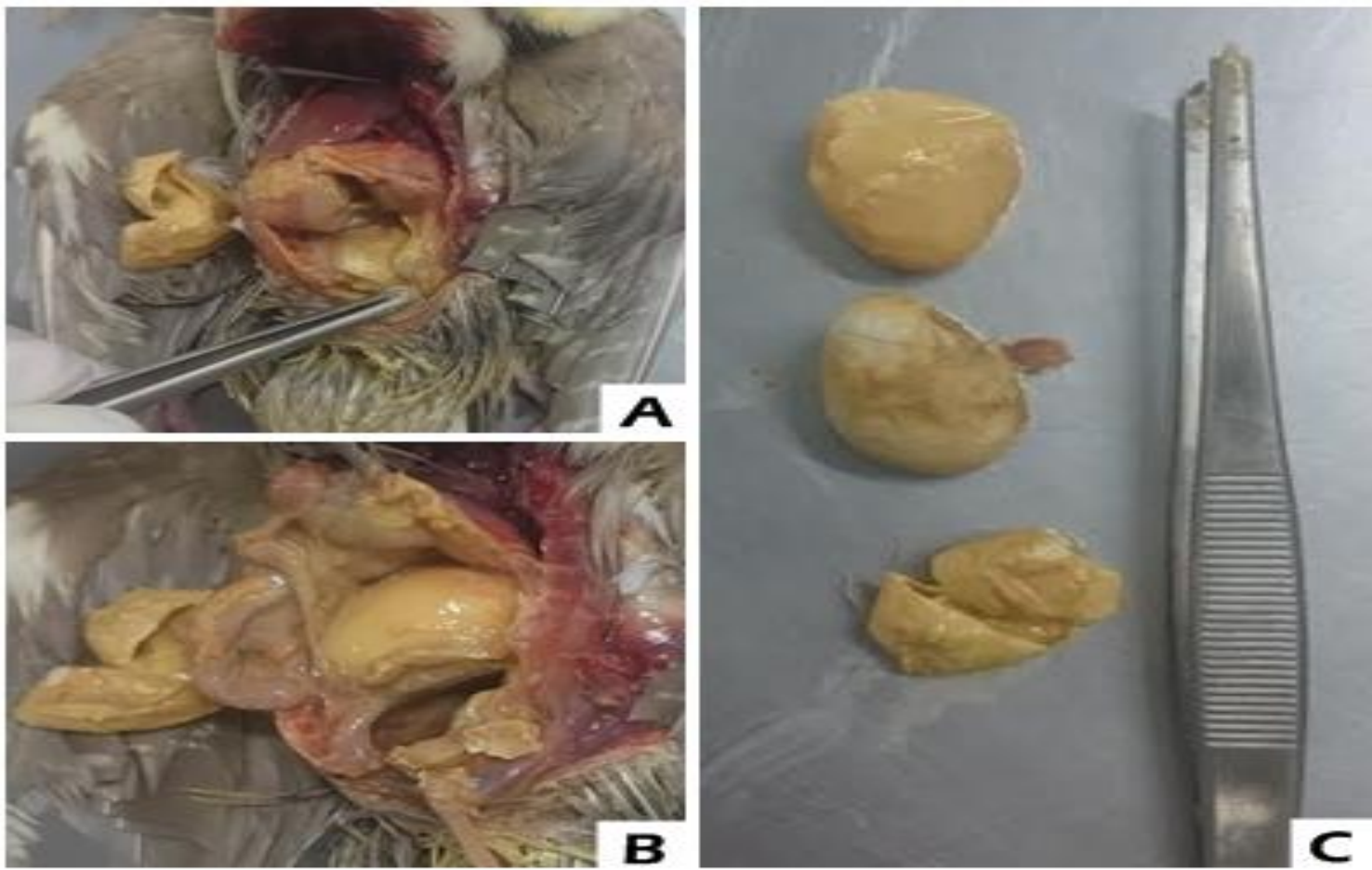

Figura 3. A) Presença de ovos livres na cavidade celomática; B) Aderência intestinal ao ovo médio; C) Três ovos, sendo apenas um com a casca totalmente mineralizada.

\section{Resultados e discussão}

As evidências clínicas obtidas a partir da anamnese e exame físico, associado ao resultado do exame de radiografia, o qual permitiu a localização do ovo, foram suficientes para o fechamento do diagnóstico de distocia como relatado na literatura (Rosen, 2012). 
A retenção de ovo como no relato representa uma de várias patologias que acomete aves em clínica veterinária. Santos et al. (2008) observaram que 253 aves selvagens atendidas na Universidade Federal do Paraná num período de quatro anos de estudos, apenas 1,04\% apresentavam quadro de retenção. Esta patologia pode ser ocasionada por múltiplos fatores, dos quais se cita a deficiência nutricional (Hasholt, 1966), uma vez que, a base da alimentação do paciente atendido consistia de sementes, principalmente de girassol. Outro fator favorável seria a predisposição genética de distocia na ave (Marietto Gonçalves et al., 2007), visto que a mesma apresentou quadro de recidiva.

A ave apresentou inicialmente quadro agudo de distocia, tratada pelo proprietário através de massagem abdominal com unguento e compressa de água morna, metodologia medicamentosa eficaz em alguns quadros de distocia (Marietto Gonçalves et al., 2007). No entanto, diante de recidiva o paciente em atendimento, foi novamente tratado inicialmente com terapia medicamentosa por período de 11 dias sem sucesso, corroborando com a literatura em casos de distocia quando não há sucesso medicamentoso, se faz necessário o uso de intervenção cirúrgica (Hasholt, 1966), sendo a técnica de histerectomia o tratamento cirúrgico de primeira escolha para paciente com recidiva (Castro et al., 2013). Contudo, há risco para aves de pequeno porte, devido aos procedimentos cirúrgicos, sendo uma alternativa menos invasiva o uso de vídeo cirurgia (Pye et al., 2001).

Devido ao tempo de evolução patológica, ocorreu um acumulo de ovos na cavidade da ave, comprimindo todos os órgãos da cavidade, devido a isto, possivelmente causou isquemia renal no paciente (Bretz, 2015; Rosen, 2012), justificando a presença de excreção em grande quantidade de urato nas fezes, sendo um indicativo de comprometimento do sistema renal das aves (Bretz, 2015; Rosen, 2012). A literatura afirma que nesses casos a compressão dos nervos e vasos locais pode favorecer quadros de claudicação, paresia e ainda diminuição de perfusão sanguínea em outros órgãos, o que possivelmente pode causar necrose dos mesmos (Rosen, 2012).

Como medidas paliativas e menos invasivas foram realizadas a descompressão abdominal por ovocentese (Bretz, 2015), acrescido da administração do alopurinol via oral $(10 \mathrm{mg} / \mathrm{kg})$, com intuito de inibir o catabolismo das purinas, evitando o acúmulo maior de urato nos órgãos e articulações do paciente (Rosen, 2012).

\section{Conclusão}

Em casos de distocia associada ao tratamento tardio existe uma maior possibilidade da ocorrência de quadros de comprometimento metabólico em virtude do acúmulo de ovos na cavidade abdominal da ave, logo favorecendo a ruptura de oviduto, aderência e isquemia renal como de outros órgãos.

\section{Agradecimentos}

Agradecemos ao Hospital Veterinário Metropolitano de Caucaia - HVM, juntamente com a Faculdade Terra Nordeste - FATENE pela parceria, apoio e incentivo à pesquisa científica.

\section{Referências bibliográficas}

Bretz, B. A. M. (2015). Síndrome da gota úrica em aves mantidas em cativeiro: artigo de revisão. $N B C$ Periódico Científico do Núcleo de Biociências, 5(9):21-26.

Castro, P. F., Fantoni, D. T. \& Matera, J. M. (2013). Estudo retrospectivo de afecções cirúrgicas em aves. Pesquisa Veterinária Brasileira, 33(5):662-668.

Crosta, L., Gerlach, H., Bürkle, M. \& Timossi, L. (2003). Physiology, diagnosis, and diseases of the avian reproductive tract. The Veterinary Clinics of North America. Exotic Animal Practice, 6(1):5783.

Gündüz, M. C., Toydemir, S., Enginler, S. A., Yilmaz, O. T., Yigit, F. \& Uçmak, M. (2009). Hysterectomy operation for the treatment of the eg g binding in the lovebird (agapornis comprise): case report. İstanbul Üniversitesi Veteriner Fakültesi Dergisi, 35(1):43-48.

Hasholt, J. (1966). Diseases of the female reproductive organs of pet birds. Journal of Small Animal Practice, 7(4):313-320. 
Marietto Gonçalves, G. A., Lima, E. T., Sequeira, J. L. \& Andreatti Filho, R. L. (2007). Colisepticemia em Papagaio verdadeiro (" Amazona aestiva")-Relato de Caso. Revista Brasileira de Saúde e Produção Animal, 8(1):56-60.

Matos, R. \& Morrisey, J. K. (2005). Emergency and critical care of small psittacines and passerines. Paper presented at the Seminars in Avian and Exotic Pet Medicine.

Pye, G. W., Bennett, R. A., Plunske, R. \& Davidson, J. (2001). Endoscopic salpingohysterectomy of juvenile cockatiels (Nymphicus hollandicus). Journal of Avian Medicine and Surgery, 15(2):90-95.

Rosen, L. B. (2012). Avian reproductive disorders. Journal of Exotic Pet Medicine, 21(2):124-131.

Santos, G. G. C., Matuella, G. A., Coraiola, A. M., Silva, L. C. S., Lange, R. R. \& Santin, E. (2008). Doenças de aves selvagens diagnosticadas na Universidade Federal do Paraná (2003-2007). Pesquisa Veterinária Brasileira, 28(11):565-570.

Recebido: 10 de julho, 2019.

Aprovado: 26 de agosto, 2019.

Publicado: 17 de setembro, 2019.

Licenciamento: Este artigo é publicado na modalidade Acesso Aberto sob a licença Creative Commons Atribuição 4.0 (CC-BY 4.0), a qual permite uso irrestrito, distribuição, reprodução em qualquer meio, desde que o autor e a fonte sejam devidamente creditados. 